\title{
Research on Intelligent Water Network System Based on Multi - modal Data Fusion
}

\author{
Ru-Ying Lv, Jing Wang \\ Faculty of Information technology, \\ Beijing University of Technology, \\ Engineering Research Center of Digital Community, Beijing, China \\ E-mail: 920774190@qq.com,dkwangjing@emails.bjut.edu.cn
}

\begin{abstract}
The rapid development of the 21st century, so that the number of types and number of various types of exponential growth is not only the amount of data, large data era has come quietly. Intelligent water network system is also indispensable to large data. In the context of large data, large data analysis for the intelligent water network provides technical support. The connotation of intelligent water network is introduced briefly and this paper analyzes the use of ontology to carry on the new structure to the intelligent water network system, Finally, the future research center and the problem are described.
\end{abstract}

Keywords-intelligent water network; large data; ontology; Multimodal data fusion

\section{INTRODUCTION}

China is a country with severe drought and water shortage. Although freshwater resources account for $6 \%$ of the world's water resources, ranked fourth in the world, but China's per capita water resources is only 2300 cubic meters, only the world average of $1 / 4$, is one of the world's poorest countries per capita water resources. Coupled with the increase in water use every year, the use of water as a very important issue can not be ignored. Urban water network efficiency is becoming low, and the aging degree is more and more serious. In response to these problems, intelligent water network came into being. Intelligence is to maintain the original water network pipeline, reduce the dependence on artificial, joined the sensor technology, data mining technology, analysis and prediction model, information network, which control the water network and provide a guarantee of accuracy, For the smart water network research has done a bedding. The development of the major cities, industrial facilities, and economic zones in areas where there is little or no import of water is inseparable from water, and therefore smart water networks are essential.

Through various sensors, we collected a variety of data, the data have some differences in the type, format, semantics. Therefore, we need to carry out ontology-based data fusion for these data. Data fusion technology, also known as information fusion technology is a comprehensive summary of a variety of information in order to obtain more accurate and more user-requested content of the technology. It first appeared in the military intelligence communication system, early research and more concentrated in the military. With the intelligent industrial systems, the technology was gradually extended to civilian use. For the data collected in the intelligent water network, we consider the system architecture based on ontology fusion.

\section{THE CURRENT SITUATION OF RESEARCH}

At present, the amount of data increases at a rate of 3 times per year[1]. On the basis of water information collection, storage, management and exchange, the monitoring of water has been realized, the data sharing and application integration architecture has been preliminary completed. In the data acquisition, through the various telemetry systems, rainfall, rain and other conditions have complete data. We built a front library, the exchange business library and integrated library link between the three, so that data can eventually appear in the integrated library, which in the shared exchange platform to display it.

In the laying of the pipeline Intelligent water network project equipped with hundreds of yellow buoys, buoys with sensors. Through wireless networking, these sensors can measure water temperature, salinity and oxygen content.

Each data is gathered through the shared switching platform and stored in the corresponding table. The data is managed according to the object-relational model of the whole team, as different object attributes. According to the object relational model, the basic object data table is managed through the global object relation table. In this complete model specification, the data can be collected more standardized and intuitive management, so that the data organization, the use of efficient and convenient.

At present, the data of water network management include hydrological and meteorological data, water supply and drainage data, water resources data, work data, geographic information data, etc. Each kind of information includes real-time data and basic data. It can support the organization, search and discovery of the massive information through metadata, and realize the quick construction and standard management of the data warehouse, so that the users can discover the information resources through various retrieval ways, and achieve the goal of sharing and sharing information resources. Metadata is data about data, which is used to describe specific resource objects so that resource objects can be located, retrieved, and managed. Metadata is a structured description of information resource objects. It can integrate and integrate various kinds of complex information to help users understand the content and quality of information. 


\section{INTELLIGENT WATER NETWORK AND ONTOLOGY FUSION}

The intelligent water network is the product of the deep coupling of water physical network, water network and water dispatch management network. "Water network construction" refers to the construction of water conservancy works effective Unicom river reservoir, build decision support platform to manage various types of water conservancy facilities, water cycle control water conservation process. With the modern water management ideas and the continuous development of information technology, water network construction is working towards the direction of intelligent development.

According to the overall needs of the water work in the future society, based on the overall framework of the intelligent water network triple play, according to the functional relationship between the water physical network, water network and water transfer network, The meaning is as follows:

\section{A. Seamless, Comprehensive Perception}

An obvious feature of intelligent network is adopting the new technology, the ability to greatly enhance the collection of information, Internet of things, 3S, cloud computing technology is fully used in the integrated management of urban water. Data acquisition coverage area has greatly improved, basically covering all aspects of the water cycle system.

\section{B. Open, Integrated Data Management}

The data collected is very much need to build an efficient and open data management structure, further integration of data storage, management, application in various links. Through the supercomputer and cloud computing integrate "water things networking", complete digital city water facilities and physical city water seamless integration. With the emergence of new data sources, data integration methods also progress continuously, followed by the emergence of XML-based data integration [2, 3], based on P2P technology data integration [4], based on Web Service technology Data integration [5] and so on.

\section{Efficient and Convenient Application Support}

Ultimately needed to show up, at this time the application support is very important to the e-government, water business management, information monitoring, water security and other fields and needs to provide intelligent support and management, to provide personalized services to the public.

Multi-modal data fusion, mainly refers to the use of computers for a variety of modal information processing, put forward a variety of theories and algorithms for different types of multi-modal data processing, in order to obtain a more accurate objective state And environmental information, is the focus of multimodal data fusion research. The research focuses on feature recognition and fusion algorithms, which can coordinate and complement different sensor information, improve the decision process based on uncertain data, and can solve the problem that can not be determined by common methods. In multimodal fusion systems, the data measured by the various sensors will have different characteristics and be expressed in different formats. Multi-modal data fusion system will make full use of the resources provided by a variety of sensors, through a variety of data reasonable analysis and utilization of these data in accordance with prior optimization principles to coordinate together to get the final fusion results, observation objectives of the state or activity rule to judge and explain. Compared with a single sensor system, multi-mode sensor system has the following advantages: the system stability, wide coverage, all the time have information to provide high reliability, information uncertainty, high spatial resolution, choose flexible.

\section{RESEARCH ON INTELLIGENT WATER NETWORK PLANNING}

\section{A. Multimodal Data Fusion System}

In distributed data integration, there are two heterogeneous cases, one is structural isomerism, and the other is semantic heterogeneous. The structural heterogeneity in intelligent water network refers to the different data structures stored in different systems. The semantic heterogeneity refers to the difference between the contents and meanings of the data items in the water network. Metadata in the history of data integration occupies a certain position. In the process of data integration, only the use of metadata alone will be limited, so the need to integrate with the specific domain ontology, including metadata in the metadata more semantic metadata, then the metadata-based resource integration architecture as shown in Figure 1.

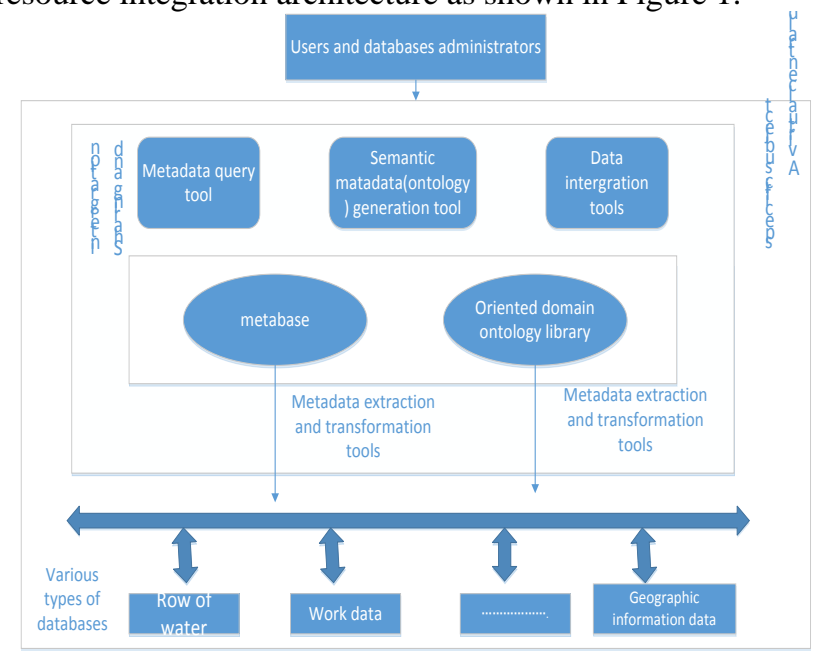

Figure 1.

The metadata-based resource integration architecture.

\section{B. Application Architecture of Water System}

The water data service currently uses a distributed and centralized organizational structure. In the service center to provide the core of centralized data services, the following branches of the core data generated together to form a centralized management of data integration, and then through a unified external data interface to provide data 
services. Between the branches, they share core data and then distributed to build their own applications, including hardware platforms and databases and application systems and other software platforms. Distributed file system refers to the management of the file system's physical storage is not all connected with the local node, but with the computer network and node connectivity. Many companies now have their own distributed file systems, such as Taobao File System (TFS) from Taobao, GFS (Google File System) from Google, Cosmos from Microsoft, GPFS (General Parallel File System) from IBM and The Alibaba Distributed File System (ADFS) for Alibaba.

With the increase of the application system, the complexity and efficiency of the system decrease gradually, and the problems of repeated construction and decentralization of each branch are exposing gradually, which have great negative effects on the system application scale and system maintenance.

\section{Unified Water Network Data Analysis System}

Water network data contains many types of basic information, spatial information, sensor observation information, business information of each system and external access to shared data, while the original data value density is low, with typical " $4 \mathrm{~V}$ " characteristics, they are scale Such as volume, variety, velocity and value [6] or veracity [7]. In traditional information processing means to show more and more limitations. Large data technology is considered to be a powerful tool to solve the abovementioned major problems. The processing of large data can be abstractly understood as data extraction, cleanup, loading process and data mining and knowledge discovery process.

The process of extraction, cleanup and loading is to complete the data format conversion, fault tolerance and information enrichment and other pretreatment work, formate a unified data model under the management of the standard data. In which the data management system by adding large data technology, it can effectively enhance the coverage and processing power for the upper analysis and decision-making and post-work to provide a more comprehensive and in-depth information service capabilities.

\section{Unified Water Network Cloud Service System}

Water comprehensive data will be fully integrated water survey data and spatial data, the establishment of the location characteristics of the water space database, in the future can be used to complete the water information network construction mode. The data, hardware platform, database software, and the core application system unified construction. Based on cloud computing software and service model, for all users, to provide targeted, various forms of information dissemination, visualization level of integrated information services. Form an integrated, flexible water cloud data center architecture. Cloud computing data center architecture is showed as Figure 2.

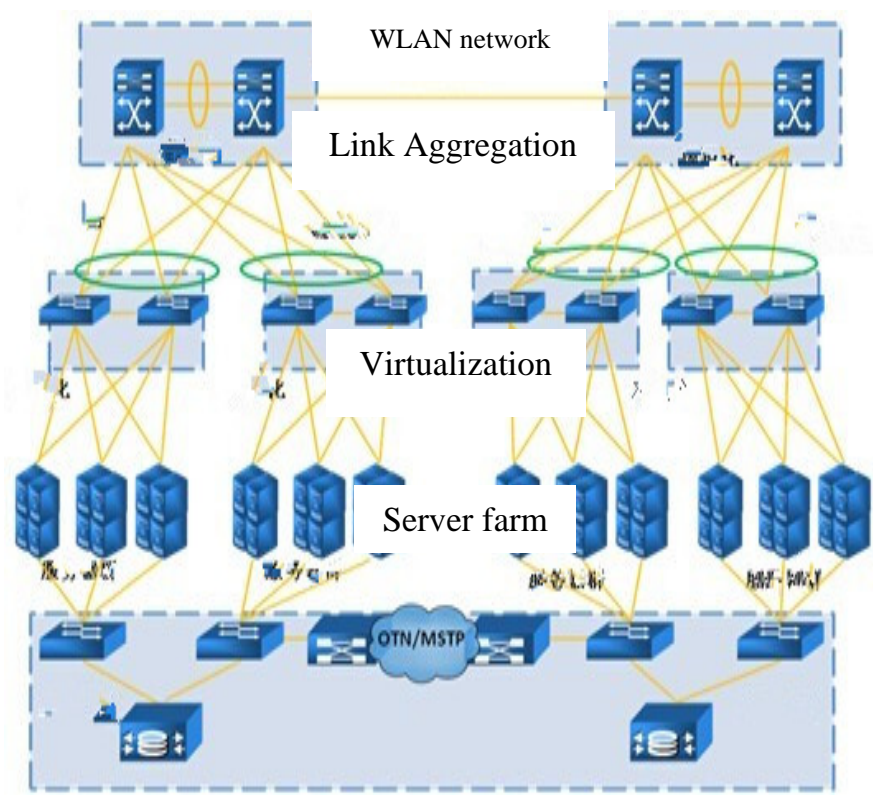

Figure 2. Cloud computing data center architecture.

\section{SUMMARY AND PROSPECT}

Based on the demand of intelligent water network users and the research of multimodal data fusion and large data technology, the intelligent water network system is further researched, and the intelligent water network system of multi-modal data fusion is presented. It provides convenient information for users and database administrators to understand the data in a more comprehensive way.

Intelligent water network to the city water users show a qualitative leap, It is the hub of information delivery, its operation and management should be based on the continuous development of business needs and development. In the future, further research on ontology technology has very important practical value.

Ontology can be understood as an area of various standard terms vocabulary and its precise definition[8], as well as the relationship between these terms. In the realization process, the ontology is composed of concept, attribute and instance.

Different information systems in the system functions, standards, technology and other aspects of the differences, so the effective integration of the data to be a great effort. There are still a lot of places to improve on the construction and integration of parallel ontology. We should make full use of the framework of large data processing to further optimize the process of ontology fusion. We also need manual participation in ontology fusion, how to further improve the automation of fusion need us further study.

\section{REFERENCES}

[1] WinterCorp.The Large Scale Data Management Experts[EB/OL].http://www.wintercorp.com

[2] Lehti P, Fankhauser P.XML data integration with OWL;Experiences and challenges[C]//Proceedings 2004 International Symposium on Applications and the Internet.IEEE, 2004:160-167 
[3] Passi K, Lane L, Madria S, et al.A model for XML Schema integration[M]//E-Commerce and Web Technologies.Springer Berlin Heidelberg, 2002:193-202

[4] Ng W S, Ooi B C, Tan K L, et al.PeerDB:A P2P-based system for distributed data sharing[C]//Proceedings 19th International Conference on Data Engineering, 2003.IEEE, 2003:633-644

[5] Zhangshi.Sharing of Heterogeneous Data Sources Based on Web Service[D]Changsha, National Defense University, 2003:12-17
[6] James M, Michael C, Brad B.Big data:The next frontier for innovation, competition, and productivity[J].The McKinsery Global Institute, 2011(5)

[7] Big data[EB/OL].[2014-03-19].http://en.wikipedia.org/wiki/Big_data

[8] Karasavas K A, Baldock R, Burger A?, Bioinformatics Integration and Agent Technology[J]. Journal of Biomedical Informatics, 2004, 37(3):205 219 